\title{
EDITORIAL
}

\section{Cardiac arrest, gender and resuscitation outcomes}

\author{
Yigal Helviz ${ }^{1}$, Marcus Ong ${ }^{2}$ and Sharon Einav ${ }^{1,3^{*}}$ (D)
}

(0) 2018 Springer-Verlag GmbH Germany, part of Springer Nature

The symptoms of death are incontrovertible. It would therefore seem safe to assume that men and women fare similarly following cardiac arrest. However, there are currently insufficient data to support this assumption throughout all the stages of the acute care process. Although the literature on this topic is not abundant, several findings are reported that require further investigation.

Women undergoing OHCA tend to be older than men and have more comorbidities [1]. Women arrest more in the privacy of their home whereas men arrest more in public locations. Accordingly, there are fewer witnessed arrests [2] and greater delays in calling for emergency medical system assistance for women [3]. The prevalence of the different presenting rhythms differs between the sexes; women present with more non-shockable rhythms than males $[1,2]$.

None of these disadvantages seem amenable to change, making it easy to assume that these are reasonable grounds for poorer outcomes among women. However, preventable causes have recently begun to emerge. Women receive less bystander CPR [4]. The interval to first rhythm recording and first chest compression is longer in women. There are more difficulties in achieving intravenous access in women than in men during OHCA. Women receive fewer per-protocol resuscitation medications [3]. However, despite all of these disadvantages, women usually have a higher rate of return of spontaneous circulation (ROSC) after OHCA [1,2,5,6].

Looking forward, assessment of the functional outcomes and quality of life of survivors at meaningful time

*Correspondence: einav_s@szmc.org.il

${ }^{1}$ Intensive Care Unit, Shaare Zedek Medical Center, POB 3235, 91031 Jerusalem, Israel

Full author information is available at the end of the article points (e.g., 3-6 months after hospital discharge) should be the end targets of any comparison between the sexes regarding resuscitation outcomes. However, multiple quality indicators are easily identifiable along the way to this final outcome. These include both interim outcome and process measures.

One traditional interim outcome measure is survival to hospital discharge. Conflicting data exist regarding the association between sex and the likelihood of discharge from hospital following OHCA-some studies show better outcomes in women $[2,6,7]$, some demonstrate similar outcomes [8-10], and some show worse outcomes compared with men $[4,11]$. Another interim outcome measure is the rate of neurologically intact survival at the time of hospital discharge. Few studies provide insight into this outcome; these show that women have outcomes that are similar to or poorer than those of men [11-16] (Table 1).

Process measures that are only beginning to be studied include the adjusted rates of provision of in-hospital interventions [e.g., targeted temperature management (TTM), coronary catheterization] and complication rates. When men and women were treated equally with TTM, neurologic outcomes as assessed by modified Rankin scales were poorer in women despite an otherwise seemingly similar physiologic response [17]. That catheterization may be underutilized in women after cardiac arrest cases is suggested by studies showing that the adjusted rates of early coronary angiography are significantly lower in women than in men [12, $15,16,18]$ although their rates and outcomes after percutaneous coronary intervention are similar [12]. Younger age, typical pre-arrest chest pain and a higher prevalence of shockable rhythms may explain clinician inclination to perform more diagnostic coronary angiographies in males, but there has been no study proving this. Similarly, no study has examined whether

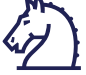 Springer}




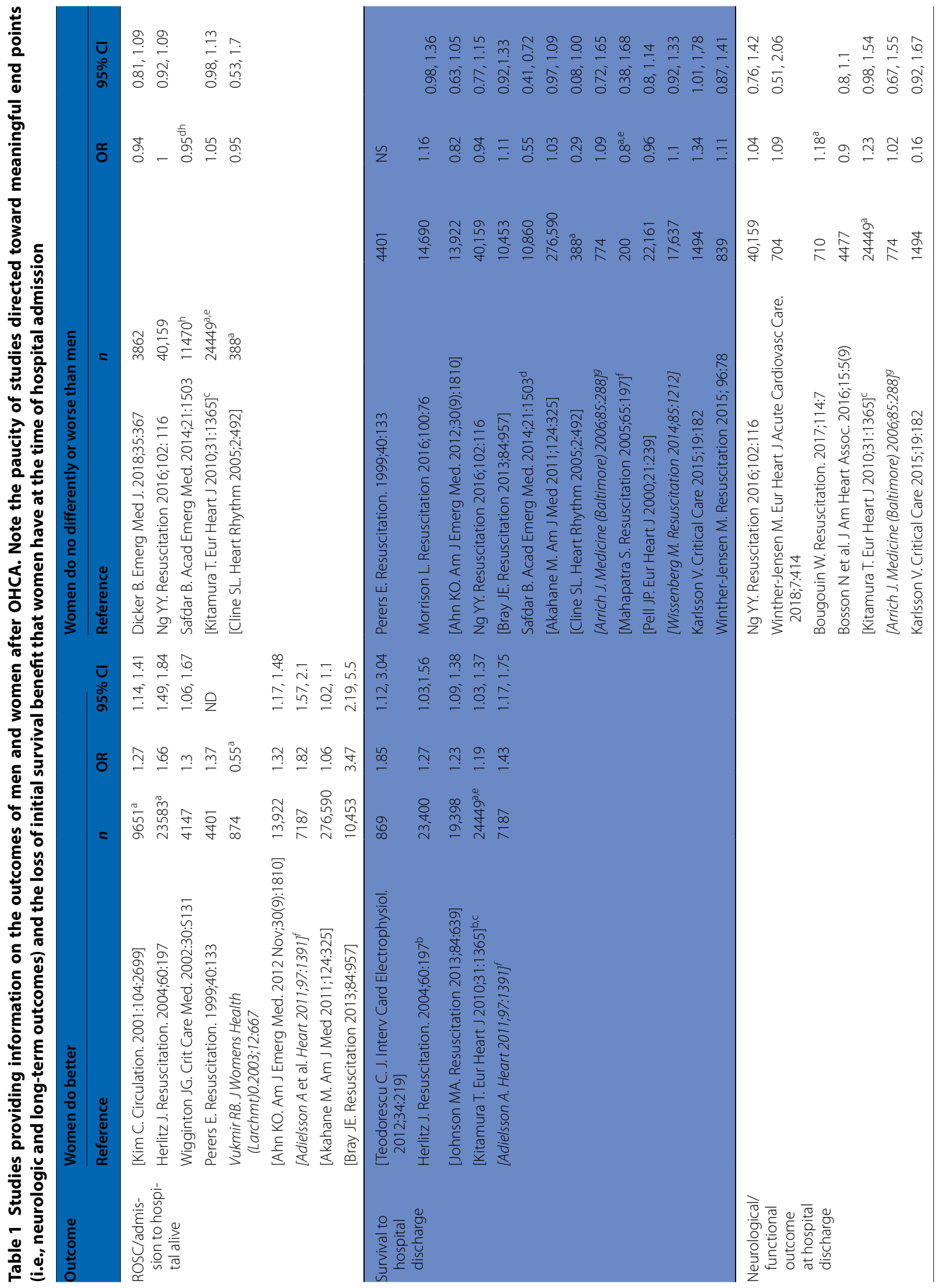


differences in catheterization rates are driven by differences in post-ROSC electrocardiography. Quality measure programs that assess the management of myocardial infarctions typically include only STEMI cases in door-to-balloon registries. Atypical presentation of CAD probably causes more arrests in women, but this has yet to be proven. Some of these women may not undergo coronary catheterization despite potential for benefit. There is also no study comparing post-ROSC echocardiography. Men generally have more systolic heart failure whereas women have more diastolic heart failure, which comprises a greater treatment challenge [19]. Whether such differences affect post-ROSC severity of heart failure, management strategies and overall resuscitation outcomes remains to be elucidated.

Conservative management may stem from concerns regarding possible treatment intolerance or lack of benefit [20]. Women are generally referred less to cardiac interventions (e.g., coronary catheterization, coronary artery bypass graft surgery), and when referred, their outcomes are worse than those observed in men undergoing the same procedures [20]. Unsurprisingly, data from the International Cardiac Arrest Registry (INTCAR) showed differences between men and women in both type and prevalence of complications occurring during ICU care after ROSC as well. However, this study was limited to patients admitted to an ICU and treated with TTM. Such selection criteria assume a priori that care has been equal at all treatment points up to the time of patient inclusion. Concerns regarding potential complications may lead to deferral of invasive interventions. However, whether selective deferral occurs, its justifications or lack thereof, and its relationship with complication rates and outcomes after ROSC remain unknown. Follow-up in the INTCAR study was also limited to the time of hospital discharge, leaving the long-term implications of these complications a topic for speculation [16].

At this time no information is available regarding patient severity and therapeutic intervention scores (e.g., APACHE, TISS), type of admitting ICU and ICU length of stay, resource consumption and rates of withdrawal/withholding of care in men and women. Are men admitted more to specialized coronary care units after ROSC specifically because these provide catheterization? Patients with shockable and non-shockable rhythms are often admitted to different ICUs. Different ICUs have different treatment approaches, which may affect patient outcomes. Many medications currently being used to treat heart disease have undergone less investigation on female subjects and have more side effects in women than in men [21]. Could these affect post-ROSC outcomes? Adjusted comparisons need to be performed to answer these questions and many more. 
To provide the best intensive care to our post-ROSC patients, more research must be invested in genderrelated aspects of the treatment they receive. Registries that do not include data on post-discharge survival, functional outcomes and quality of life should be encouraged to include such data. Those that already include data on both sex and outcome at or after hospital discharge should be investigated in greater depth. Studies must be specifically targeted to such comparisons, including adjustments for patient background diseases, arrest characteristics and post-ROSC conditions. The risk-benefit ratio of various treatments in female versus male patients requires more in-depth investigation. OHCA registries should be linked to in-hospital data on organ failure and neurologic follow-up, both of which could explain differences in treatment if such are found. If we carry on ignoring the details that we, as intensivists, know are important, we will also continue to remain ignorant of potential inequalities in the care we are delivering to our patients.

\section{Author details \\ ${ }^{1}$ Intensive Care Unit, Shaare Zedek Medical Center, POB 3235, 91031 Jerusa- lem, Israel. ${ }^{2}$ Department of Emergency Medicine, Singapore General Hospital and Duke-NUS Medical School, Health Services and Systems Research (HSSR), Singapore, Singapore. ${ }^{3}$ Faculty of Medicine, Hebrew University, Jerusalem, Israel.}

\section{Conflicts of interests}

None of the authors has any conflict of interest to disclose regarding the topic of this manuscript.

Received: 31 August 2018 Accepted: 30 November 2018

Published online: 7 December 2018

\section{References}

1. Wigginton JG, Pepe PE, Bedolla JP et al (2002) Sex-related differences in the presentation and outcome of out-of-hospital cardiopulmonary arrest: a multiyear, prospective, population-based study. Crit Care Meds 30:S131-S136

2. Teodorescu C, Reinier K, Uy-Evanado A et al (2012) Survival advantage from ventricular fibrillation and pulseless electrical activity in women compared to men: the Oregon sudden unexpected death study. J Interv Card Electrophysiol 34:219-225. https://doi.org/10.1007/s1084 0-012-9669-2

3. Mumma BE, Umarov T (2016) Sex differences in the prehospital management of out-of-hospital cardiac arrest. Resuscitation 105:161-164. https:// doi.org/10.1016/j.resuscitation.2016.05.029

4. Safdar B, Stolz U, Stiell IG et al (2014) Differential survival for men and women from out-of-hospital cardiac arrest varies by age: results from the OPALS study. Acad Emerg Med 21:1503-1511. https://doi.org/10.1111/ acem. 12540
5. Kim C, Fahrenbruch CE, Cobb LA, Eisenberg MS (2001) Out-of-hospital cardiac arrest in men and women. Circulation 104:2699-2703

6. Herlitz J, Engdahl J, Svensson L et al (2004) Is female sex associated with increased survival after out-of-hospital cardiac arrest? Resuscitation 60:197-203. https://doi.org/10.1016/j.resuscitation.2003.09.012

7. Bougouin W, Mustafic H, Marijon E et al (2015) Gender and survival after sudden cardiac arrest: a systematic review and meta-analysis. Resuscitation 94:55-60. https://doi.org/10.1016/j.resuscitation.2015.06.018

8. Perers E, Abrahamsson P, Bång A et al (1999) There is a difference in characteristics and outcome between women and men who suffer out of hospital cardiac arrest. Resuscitation 40:133-140. https://doi.org/10.1016/ S0300-9572(99)00022-2

9. Morrison LJ, Schmicker RH, Weisfeldt ML et al (2016) Effect of gender on outcome of out of hospital cardiac arrest in the resuscitation outcomes consortium. Resuscitation 100:76-81. https://doi.org/10.1016/j.resuscitat ion.2015.12.002

10. Ahn KO, Shin SD, Hwang SS (2012) Sex disparity in resuscitation efforts and outcomes in out-of-hospital cardiac arrest. https://doi.org/10.1016/j. ajem.2012.02.018

11. Ng YY, Wah W, Liu N et al (2016) Associations between gender and cardiac arrest outcomes in Pan-Asian out-of-hospital cardiac arrest patients. Resuscitation 102:116-121. https://doi.org/10.1016/j.resuscitat ion.2016.03.002

12. Bougouin W, Dumas F, Marijon E et al (2017) Gender differences in early invasive strategy after cardiac arrest: Insights from the PROCAT registry. Resuscitation 114:7-13. https://doi.org/10.1016/j.ressuscita tion.2017.02.005

13. Bosson N, Kaji AH, Fang A et al (2016) Sex differences in survival from out-of-hospital cardiac arrest in the era of regionalized systems and advanced post-resuscitation care. J Am Heart Assoc 5:e004131. https:// doi.org/10.1161/JAHA.116.004131

14. Hagihara A, Onozuka D, Hasegawa M et al (2017) Resuscitation outcomes of reproductive-age females who experienced out-of-hospital cardiac arrest. Eur Heart J Acute Cardiovasc Care 6:121-129. https://doi. org/10.1177/2048872616633879

15. Winther-Jensen M, Hassager C, Kjaergaard J et al (2018) Women have a worse prognosis and undergo fewer coronary angiographies after outof-hospital cardiac arrest than men. Eur Heart J Acute Cardiovasc Care 7:414-422. https://doi.org/10.1177/2048872617696368

16. Karlsson V, Dankiewicz J, Nielsen N et al (2015) Association of gender to outcome after out-of-hospital cardiac arrest-a report from the international cardiac arrest registry. Crit Care 19:182. https://doi.org/10.1186/ s13054-015-0904-y

17. Winther-Jensen M, Kjaergaard J, Wanscher M et al (2015) No difference in mortality between men and women after out-of-hospital cardiac arrest. Resuscitation 96:78-84. https://doi.org/10.1016/j.resuscitat ion.2015.06.030

18. International Cardiac Arrest Registry (INTCAR) |. https://www.intcar.org/. Accessed 27 Oct 2018

19. Lenzen MJ, Rosengren A, op Reimer WJMS et al (2008) Management of patients with heart failure in clinical practice: differences between men and women. Heart 94(3):e10.https://doi.org/10.1136/hrt.2006.099523

20. Johnston N, Bornefalk-Hermansson A, Schenck-Gustafsson K et al (2013) Do clinical factors explain persistent sex disparities in the use of acute reperfusion therapy in STEMI in Sweden and Canada? Eur Heart J Acute Cardiovasc Care 2:350-358. https://doi.org/10.1177/2048872613496940

21. Vitale C, Fini M, Spoletini I et al (2017) Under-representation of elderly and women in clinical trials. Int J Cardiol 232:216-221. https://doi. org/10.1016/j.ijcard.2017.01.018 